Anaesthesist 2021 · 70:420-431

https://doi.org/10.1007/s00101-020-00890-8

Online publiziert: 27. November 2020

(C) Der/die Autor(en) 2020

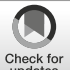

Janosch Dahmen ${ }^{1,2,3} \cdot$ Linnart Bäker ${ }^{1}$. Florian Breuer ${ }^{1,2} \cdot$ Karsten Homrighausen $^{1}$. Christopher Pommerenke ${ }^{1} \cdot$ Jan-Karl Stiepak ${ }^{1,2} \cdot$ Stefan Poloczek ${ }^{1,2}$

'Berliner Feuerwehr, Berlin, Deutschland

${ }^{2}$ Ärztliche Leitung Rettungsdienst im Land Berlin, Berlin, Deutschland

${ }^{3}$ Fakultät für Gesundheit, Department Humanmedizin, Universität Witten/Herdecke, Witten, Deutschland

\title{
COVID-19-Stresstest für die Sicherstellung der Notfall- versorgung: Strategie und Maßnahmen der Notfallrettung in Berlin
}

\section{Zusatzmaterial online}

Die Online-Version dieses Beitrags (https:// doi.org/10.1007/s00101-020-00890-8) enthält weiteres Online-Zusatzmaterial.

Beitrag und Zusatzmaterial stehen Ihnen auf www.springermedizin.de zur Verfügung. Bitte geben Sie dort den Beitragstitel in die Suche ein, das Zusatzmaterial finden Sie beim Beitrag unter „Ergänzende Inhalte".

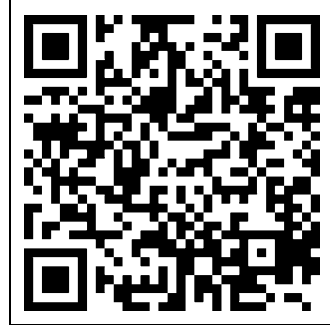

\section{Ausgangslage}

Am 05.01.2020 wurde die Ärztliche Leitung des Rettungsdienstes der Berliner Feuerwehr im Land Berlin (ÄLRD) über das Frühwarnsystem des European Emergency Medical Services Leadership Network (EMS Leadership Network) [1] und die WHO auf eine neuartige, infektiöse Lungenerkrankung in der Stadt Wuhan aufmerksam [2]. Am selben Tag berichtete die internationale Presse erstmals über 59 bekannte Infektionsfälle, davon 11 kritisch erkrankte Menschen mit einer bilateralen, aty- pischen Pneumonie in der genannten zentralasiatischen Region Hubei [3]. In den ersten Berichten gingen die verantwortlichen Stellen der Wuhan Municipal Health Commission zu diesem Zeitpunkt noch davon aus, dass eine Mensch-zuMensch-Übertragung unwahrscheinlich sei, die erste Infektion sich voraussichtlich am 12.12.2019 zugetragen habe und bisher keine Ansteckungen unter dem Personal des Gesundheitswesens in der Region festzustellen worden sei.

Vor dem Hintergrund der Erfahrungen des epidemiologischen Managements des „severe acute respiratory syndrome“ (SARS) 2002/2003 und des "middle east respiratory syndrome“ (MERS) 2012 wurden mit Bekanntwerden der Entwicklungen durch die ÄLRD als erste Maßnahmen die enge Beobachtung der epidemiologischen Entwicklung, insbesondere respiratorischer Erkrankungen, und die spezifische kontinuierliche Abstimmung mit den Partnern des EMS Leadership Network veranlasst.

Aufgrund der schnellen Ausbreitung der Infektion in den folgenden Wochen wurde am 20.01.2020 eine erste Surveillance-Arbeitsgruppe unter Leitung der Oberärztin bzw. des Oberarztes vom Dienst (OAvD) zur Identifikation notwendiger Handlungsfelder im Umgang mit COVID-19-Patienten durch den ÄLRD initiiert.

Durch die Arbeitsgruppe wurde neben der täglichen Lagedarstellung auch am 24.01.2020 eine erste „standard operating procedure" (SOP) für den Umgang mit potenziell infizierten Patientinnen und Patienten im Rettungsdienst veröffentlicht. Am 28.01.2020 wurde dann die erste Infektion in Deutschland in Bayern bei einem Patienten nachgewiesen [4]. Es folgte eine sehr dynamische und noch andauernde epidemiologisch bedeutsame Lage, die als andauernder Einsatz das gesamte Gesundheitswesen und insbesondere auch die Notfallrettung bis zum heutigen Tag herausfordert.

Der Artikel gibt vor dem Hintergrund der derzeit diskutierten Reform der Notfallversorgung in Deutschland einen Überblick über die Strategie und die bislang umgesetzten Maßnahmen der Berliner Feuerwehr und der Ärztlichen Leitung Rettungsdienst im Land Berlin im Umgang mit der andauernden SARSCoV-2-Pandemie. Aus den dargestellten Erkenntnissen werden die Bedeutungen eines umfassenden Sicherstellungsbegriffs, der klaren Zuständigkeit in der medizinischen Gefahrenabwehr resp. Notfallversorgung und auch von sektorenübergreifenden Versorgungskonzepten vor dem Hintergrund der genannten Gesetzesreform deutlich. 
Tab. 1 Durchschnittliche Einsatzdauer mit und ohne Alarmstichwort-Zusatz „akute respiratorische Erkrankung [ARE.]"

\begin{tabular}{lllll}
$\begin{array}{l}\text { Alarm- } \\
\text { stichwort }\end{array}$ & $\begin{array}{l}\text { Mit/ohne } \\
\text { ARE. }\end{array}$ & $\begin{array}{l}\text { Einsatzdauer in } \text { min } \\
\text { (Mean) }\end{array}$ & SD & $\begin{array}{l}\text { Einsatzdauer in min } \\
\text { (Median) }\end{array}$ \\
\hline NA & Mit ARE & 86,4 & $\pm 39,8$ & 76,2 \\
NA & Ohne ARE & 72,6 & $\pm 35,4$ & 69,4 \\
\hline NOTF & Mit ARE & 69,2 & $\pm 28,1$ & 65,9 \\
NOTF & Ohne ARE & 58,8 & $\pm 26,9$ & 56,4 \\
NT/NTD & Mit ARE & 64,9 & $\pm 28,3$ & 60,6 \\
\hline NT/NTD & Ohne ARE & 62,3 & $\pm 34,1$ & 57,3 \\
Sonstige & Mit ARE & 88,6 & $\pm 51,4$ & 80,8 \\
\hline
\end{tabular}

\section{Aufbau eines Pandemiestabs}

Zur Bewältigung der ab Anfang Februar sehr dynamisch anwachsenden Pandemielage wurde durch die Behördenleitung der Berliner Feuerwehr der Einsatzplan für ,epidemisch bedeutsame Lagen“ aktiviert und ein Pandemiestab mit rund 15 festen Funktionen eingerichtet. Der Stab wurde im 4-Schicht-System mit getrennten Kohorten und kontaktlosen Übergaben organisiert. Hierbei erfolgte die Übergabe mittels einer Videokonferenz; weiterhin waren Eingang und Ausgang zum Stabsraum getrennt voneinander, und die jeweiligen Stabsgruppen konnten sich aufgrund eines Zeitversatzes nicht im Stabsraum begegnen. Zur Verstärkung wurden im Verlauf in einem erweiterten Stabszellenkonzept Organisationseinheiten der Linienorganisation der Berliner Feuerwehr zu sog. 24/7Stabszellen ertüchtigt und eine dezentrale Rund-um-die-Uhr-Verfügbarkeit aller kritischen Arbeitsprozesse insbesondere auch jenseits des Einsatzgeschehens hergestellt. Außerhalb des Einsatzbetriebes, des Pandemiestabs und der angegliederten Stabszellen wurde sämtliche rückwärtige Arbeit über digitale Hilfsmittel überwiegend auf Heimarbeitsplätze verlagert.

\section{Überwachung des Ein- satzgeschehens und Darstellung besonderer Einsatzschwerpunkte}

Über den gesamten Zeitraum, bereits von der Frühphase der epidemiologi- schen Entwicklung ausgehend, stellte das Einsatzgeschehen letztlich indirekt ein immanentes, infektiologisches Frühwarnsystem durch qualitative und quantitative Veränderungen im Anrufverhalten und im Einsatzgeschehen da. Die Berliner Feuerwehr koordiniert in einer zentralen Leitstelle für das Land Berlin alle Hilfeersuchen, die über den Notruf 112 eingehen. Im Jahresmittel gehen hier je 24h 2565 Notrufe ein, aus denen im Mittel 1271 Einsätze generiert werden. In den Monaten März/April kam es wiederholt zu über 4000 Notrufen (im Mittel 3523) und bis zum Beginn des sog. Lockdowns auch zu einer deutlichen Zunahme von Einsätzen, bei zeitgleicher Zunahme der Einsatzdauer in Teilen des Einsatzgeschehens (•Tab. 1).

Sämtliche Einsätze in der Leitstelle werden unter Anwendung einer standardisierten Notrufabfrage abgefragt. Strukfragesysteme sind in Deutschland bislang nicht flächendeckend etabliert [5]; die Verwendung international ist deutlich höher.

Im Ergebnis der standardisierten Notrufabfrage wird in Abhängigkeit des ermittelten Leitsymptoms oder der geschilderten Beschwerden einer von 4608 „dispatch codes" generiert, an die wiederum spezifische Reaktionen in der Alarm- und Ausrückeordnung angebunden sind. In der Frühphase der Pandemie wurde am 26.02.2020 mit dem Ziel der Surveillance und Triage das Epidemieprotokoll des tem (AMPDS Protokoll 36 Pandemie/ Epidemie/Ausbruch) aktiviert [6, 7]. Soturierte oder standardisierte NotrufabAdvanced Medical Priority Dispatch Sys- mit bestanden die Möglichkeiten der Surveillance des Ausbruchsgeschehens, der besseren Einsatzsteuerung und insbesondere Sensibilisierung der Einsatzkräfte, einen Alarmstichwort-Zusatz „[ARE.]“ für akute respiratorische Erkrankung an das Einsatzstichwort anzubinden.

Bis zum 01.09.2020 wurden insgesamt 17.057 Einsätze mit dem Protokoll 36 abgefragt. Im Schnitt dauerten die standardisierte Notrufabfrage mit Einstiegsund Schlüsselfragen über alle Hauptbeschwerden 1:10 min, die Abfrage des Pandemieprotokolls 36 durchschnittlich zusätzlich 1:36 min. Seit Beginn der Pandemie sind bis September 2020 so allein 386 h zusätzliche Notrufabfragezeit angefallen.

Bei $11 \%$ der Gesamteinsätze wurden im Zeitraum März-Juni 2020 Einsatzmittel zu hilfsbedürftigen Menschen mit Verdacht auf eine akute respiratorische Erkrankung (•Abb. 1) geschickt. Die Einsätze mit einem Alarmstichwort-Zusatz [ARE.] dauerten im Schnitt über alle Alarmstichworte im Mittel (Median) 17 min länger als Einsätze ohne Alarmstichwort-Zusatz [ARE.] (•Tab. 1), wobei Einsätze mit einem Notarzt (NA + ARE) im Durchschnitt +14 min länger dauerten als alle übrigen Einsätze eines Notarztes, gefolgt von Einsätzen eines RTW zu einem Notfall $(\mathrm{NOTF}+\mathrm{ARE})+10 \mathrm{~min}$ im Vergleich $\mathrm{zu}$ gleichen Einsätzen ohne ARE-Zusatz. Der geringste Unterschied zeigte sich bei Notfalltransporten (NT + ARE) mit einer durchschnittlich zusätzlichen Dauer +3 min. Allein im Monat April 2020 fielen bei gleichen Alarmstichworten insgesamt zusätzlich über $900 \mathrm{~h}$ der verlängerten Einsatzdauer bei AREEinsätzen an.

Bei jedem Einsatz zu einem AREAlarmstichwort erfolgte von der Einsatzstelle anschließend eine standardisierte Kurzlagemeldung durch die Einsatzkräfte in Analogie zur RKI-Falldefinition (Zusatzmaterial online: „Falldefinitionen und Kurzlagemeldungen in Analogie zur RKI-Falldefinition“; s. Hinweis am Anfang des Artikels).

Die Anmeldung von ARE-Patienten im Krankenhaus erfolgte für alle Einsätze zentral über die Plattform für den 
Anaesthesist 2021 · 70:420-431 https://doi.org/10.1007/s00101-020-00890-8

(c) Der/die Autor(en) 2020

J. Dahmen · L. Bäker · F. Breuer · K. Homrighausen · C. Pommerenke · J.-K. Stiepak · S. Poloczek

\section{COVID-19-Stresstest für die Sicherstellung der Notfallversorgung: Strategie und Maßnahmen der Notfallrettung in Berlin}

\section{Zusammenfassung}

Hintergrund. Die COVID-19-Pandemie

stellt die Notfallversorgung in Deutschland

vor eine beispiellose Belastungsprobe.

Neben der klinischen Notfallversorgung

in den Notaufnahmen der Krankenhäuser

kommt der präklinischen Notfallrettung

die entscheidende Sicherstellungsaufgabe

notfallmedizinischer Gesundheitsversorgung

zu. Die Berliner Feuerwehr zeigt in dem vorliegenden Beitrag für die Notfallrettung im Land Berlin neue Wege auf, dieser gewachsenen Verantwortung in der prähospitalen Patientenversorgung gerecht zu werden. Methode. Es erfolgte eine systematische Darstellung der Herausforderungen und konzeptionellen Antworten der präklinischen Notfallmedizin auf die COVID-19-Pandemie am Beispiel der Notfallrettung im Land Berlin.
Ergebnisse. Die Berliner Feuerwehr koordiniert in einer zentralen Leitstelle für das Land Berlin alle Hilfeersuchen des Notrufs 112. Je $24 \mathrm{~h}$ gehen im Mittel 2565 Notrufe ein, aus denen 1271 Einsätze generiert werden. Im Rahmen der Pandemie kam es zu einer deutlichen Zunahme an Einsätzen zu Patienten mit akuten respiratorischen Erkrankungen (ARE). So erfolgten $11 \%$ der Einsätze zu Patienten mit dem Verdacht einer COVID-19-Erkrankung. Die Notrufdauer verlängerte sich bei ergänzender Abfrage des "Pandemie-Protokolls" im Schnitt um 1:36 min, die Einsatzdauer bei Einsätzen mit Alarmstichwort-Zusatz „ akute respiratorische Erkrankung [ARE.]" im Mittel um 17 min. Schlussfolgerung. Die andauernde Pandemie macht deutlich, dass Aufgaben und
Verantwortung der öffentlichen Träger der Notfallrettung über die unmittelbare, medizinische Gefahrenabwehr für Leib und Leben hinausgehen. Neben einer Lotsenund Triage-Funktion in der integrierten Leitstelle der Berliner Feuerwehr (112) konnte durch umfassende Maßnahmen der Lageüberwachung, Lagedarstellung und Lagebewältigung trotz Ausfall alternativer, ambulanter Versorgungsangebote u.a. im Bereich der Haus- und Facharztpraxen die Notfall- resp. Gesundheitsversorgung sichergestellt werden.

Schlüsselwörter

Berliner Feuerwehr · Pandemie - Rettungsdienst - Corona $\cdot$ SARS-CoV-2

\section{COVID-19 Stress test for ensuring emergency healthcare: strategy and response of emergency medical services in Berlin}

\section{Abstract}

Background. The COVID-19 pandemic represents an unprecedented severe test for emergency medicine in Germany. In addition to in-hospital emergency medicine, prehospital emergency medicine has the decisive task of fully guaranteeing emergency medical healthcare. In this article the Berlin Fire Brigade shows new ways for emergency medical services to fulfil these increased responsibilities during the pandemic in prehospital emergency medicine in the State of Berlin.

Methods. A systematic presentation of the challenges and conceptional responses of preclinical emergency medicine to the COVID19 pandemic was carried out using the example of the emergency medical services in the State of Berlin.
Results. The Berlin Fire Brigade has a dispatch center that coordinates all requests for assistance in the State of Berlin over the emergency telephone number 112 . On average a total of 2565 emergency calls are received every $24 \mathrm{~h}$, from which 1271 missions are generated. During the pandemic there was a striking increase in missions to patients with acute respiratory diseases (ARD). Of the missions $11 \%$ were carried out to patients with the suspicion of COVID-19. The duration of the emergency calls was extended on average by 1:36 min due to the additional questions in the pandemic protocol and the duration of the mission by an average of $17 \mathrm{~min}$ with the additional alarm keyword acute respiratory disease (ARD).

Conclusion. The continuing pandemic reveals that tasks and responsibilities of public services in emergency rescue go far beyond the immediate medical prevention of danger to life and limb. In addition to the controller and triage functions in the integrated dispatch center of the Berlin Fire Brigade (112), the emergency and healthcare measures could be ensured. This was accomplished by comprehensive measures for situation control, situation reports and mastering situations despite the lack of alternative outpatient care options, especially in the areas of general practitioner, public health care and medical specialist practices.

\section{Keywords}

Berlin Fire Brigade · Pandemic - Emergency medical services - Corona - SARS-CoV-2 interdisziplinären Versorgungsnachweis https://www.ivena.de.

Insbesondere zu Beginn der epidemiologischen Entwicklungen wurde die Leitstelle mit einer überdurchschnittlich großen Anzahl reiner Informationsersuchen konfrontiert, bei denen kein medizinisches Hilfeersuchen in Anlehnung an das Rettungsdienstgesetz Berlin mit originärer Zuständigkeit der Berliner Notfallrettung erkennbar war. Im Kern drehte sich der überwältigende Teil dieser Anrufe um Fragen zu Testmöglichkeiten und der Erreichbarkeit des Gesundheitsamts sowie von Hausärzten. Nach Einrichtung einer landesweiten COVID-19-Informationshotline der Senatsverwaltung für Gesundheit, Pflege und Gleichstellung wurden deshalb die Mitarbeitenden der Leitstelle für eine schnelle Differenzierung zwischen Informations- und Hilfeersuchen sensibilisiert und wurden zur Weiterleitung reiner Informationsersuchen an die COVID-19Informationshotline befähigt.

Zur Verstärkung im Aufgabengebiet der Notrufannahme wurden in einem 


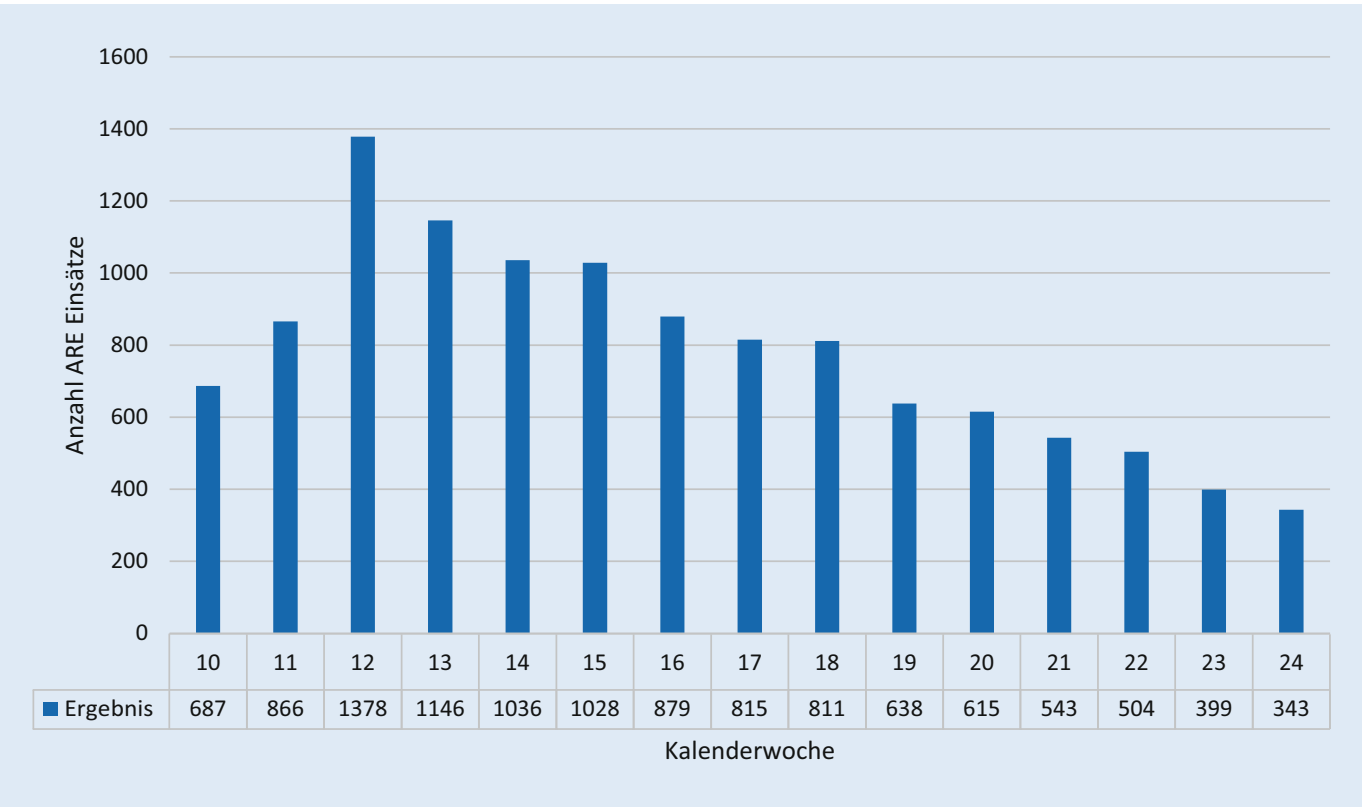

Abb. $1<$ Einsatzaufkommen mit AlarmstichwortZusatz akute respiratorische Erkrankung "[ARE.]"
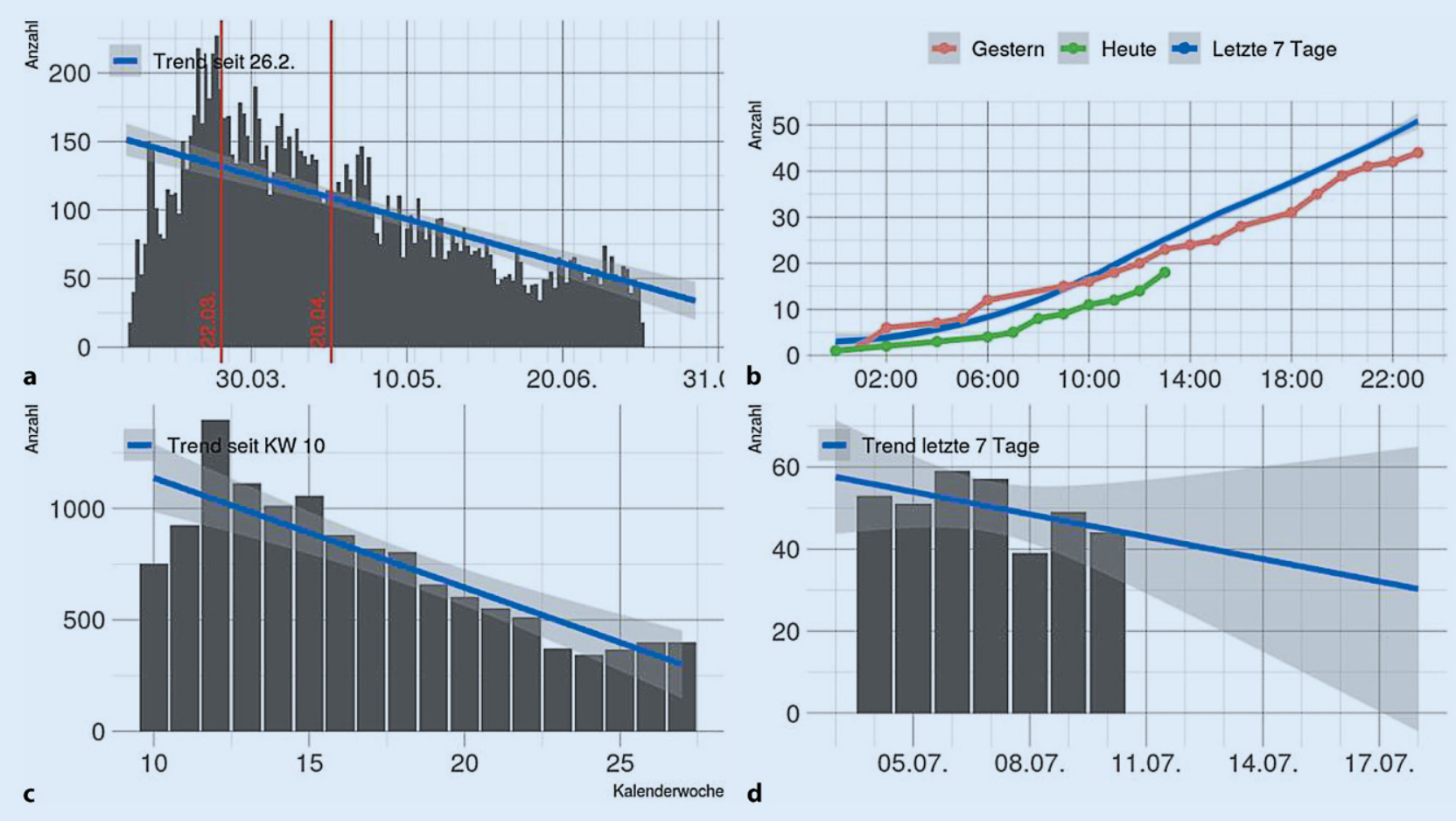

Abb. 2 A Live-Daten zum aktuellen COVID-19-spezifischen Einsatzaufkommen in der FIREAPP (IOS, Applikation, Fa. BananaGlue, Berlin). a ARE.-Einsätze/Tag, b ARE.-Einsätze, Tagesverlauf kumuliert, c ARE.-Einsätze/Woche, d ARE.-Einsätze/Tag (letzte 7 Tage). (Mit freundlicher Genehmigung der Berliner Feuerwehr). (ARE Akute Respiratiorische Erkrankung)

14-tägigen Kurs 23 Auszubildende zum Notfallsanitäter der Berliner Feuerwehr und Rettungsdienst Akademie (BFRA) unter enger Aufsicht des Qualitätsmanagements der Leitstelle qualifiziert und so die Leitstelle von 25 Funktionen (Einsatzkräften) im Tagdienst auf 31 Funktio- nen aufgestockt. Darüber hinaus wurde die bereits vor der Lage implementierte elektronische Schnittstelle zum bidirektionalen, digitalen Austausch von Hilfeersuchen der Leitstelle zum Ärztlichen Bereitschaftsdienst der kassenärztlichen Vereinigung (KV) ausgeweitet, um nach standardisierter Notrufabfrage eine Weitergabe von Anrufen zu ermöglichen. Seit der Aktivierung des Protokolls 36 wurden so 7397 niedrigprioritäre medizinische Hilfeersuchen der 112 an die Leitstelle der KV übermittelt, in Spitzenzeiten bis zu 188 Weiterleitungen am Tag. Mehrfach 


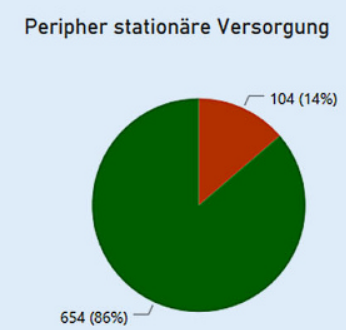

Beatmung

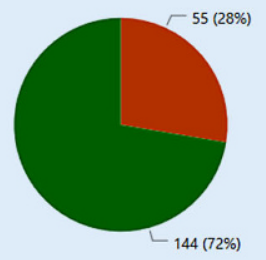

Intensivmedizinische Versorgung

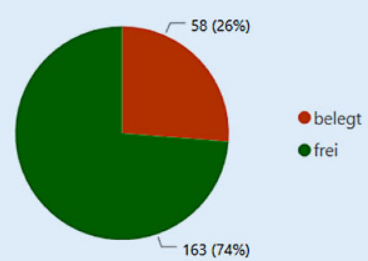

Versorgung mit ECMO

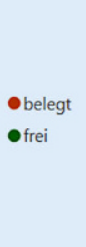

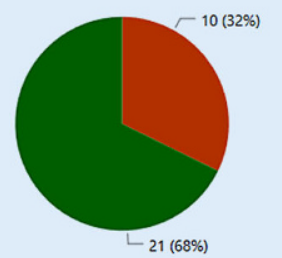

belegt

- frei
Belegt COVID-19 Frei COVID-19
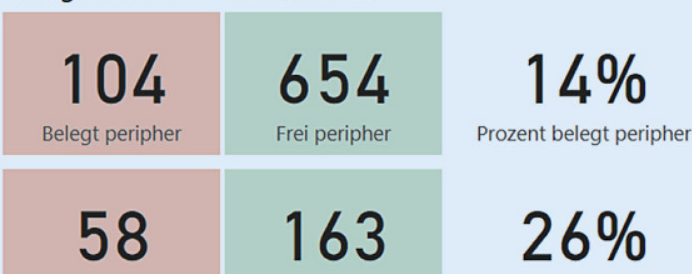

Frei intensiv

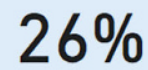

Prozent belegt intensiv

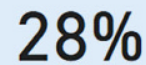

Prozent belegt Beatmung

davon belegt Beatm...

144

davon frei Beatmung

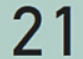

davon frei ECMO

Anzahl Krankenhäuse

Erstellt: Stab RD Datenquelle: IVENA

Abb. $3 \Delta$ VOST-Dashboard zur Darstellung der COVID-19-Klinikkapazitäten. (Mit freundlicher Genehmigung der Berliner Feuerwehr). (VOST Virtual Operations Support Team)

konnten über mehrere Stunden aufgrund der großen Anzahl an Hilfeersuchen in der Leitstelle der KV keine Anrufe über die gemeinsame Schnittstelle weitergeleitet werden und mussten hier im Ersatz mit Einsatzmitteln der Berliner Notfallrettung bearbeitet werden.

Mit dem Aufwachsen der epidemiologischen Lage wurde in der Berliner Feuerwehr ein „virtual operations support team" (VOST) implementiert. Ziel dieses Teams war es, die Lagebeurteilung und Entscheidungsfindung durch IT-gestützte Analysen und Visualisierungen $\mathrm{zu}$ unterstützen. Eine weitere Aufgabe des VOST war die Entwicklung von Ad-hoc-IT-Lösungen, beispielsweise Miniapplikationen zur Lagerbestandsüberwachung oder für die Betreuung der Mitarbeiterhotline. Hierfür wurde ein Team von IT-Spezialist ${ }^{\star}$ Innen aus verschiedenen Abteilungen der Berliner Feuerwehr zusammengestellt und in die Struktur des Pandemiestabes integriert. Durch die Krisenstäbe der Senatsverwaltungen des Landes Berlin wurde dem Einsatzstab der Berliner Feuerwehr die Aufgabe der zentralen, organisations- übergreifenden digitalen Lagedarstellung für das Land Berlin übertragen. Aufgabe des VOST war deshalb die kontinuierliche Darstellung der wichtigsten „key performance indicators“ (KPI) in diversen Dashboards und digitalen Anwendungen. Technologisch im Zentrum standen dabei insbesondere das Geoinformationssystem ArcGIS der Fa. ESRI, Redlands, Kalifornien, USA, Office-365Produkte der Fa. Microsoft, Redmond, Washington, USA, die App NaProt zur digitalen Einsatzberichtserstattung der Fa. Pulsation IT, Berlin, Deutschland und die Einsatzsteuerungssoftware FIRE der Fa. BananaGlue, Berlin, Deutschland. Durch die verschiedenen Anwendungen war es möglich, das der Pandemielage geschuldete spezifische Einsatzgeschehen (•Abb. 2), die Systemauslastung, einschließlich Leitstellen, KPI (Bettenzahlen, Zuweisungen, Anrufaufkommen), Klinikkapazitäten (• Abb. 3) sowie longitudinale Fallzahlentwicklung ( $\mathrm{Zu}$ satzmaterial online: „Live-Daten zum longitudinalen COVID-19-Einsatzaufkommen in der FIRE APP“, s. Hinweis am Anfang des Artikels) und Live-Ein- satzdaten (Zusatzmaterial online: „LiveDaten zu einem laufenden COVID-19Einsatz in der FIRE APP auf dem iPad des Einsatzfahrzeugs“, s. Hinweis am Anfang des Artikels) zur Darstellung zu bringen.

Besondere Bedeutung zur Identifikation von potenziellen Hotspots, beispielsweise Ausbruchgeschehen in organisierten Wohnformen, hatte dabei das Software-Tool FIRE-Analytics. Einsatzdaten wurden statistisch analysiert und COVID-19-bezogene Einsätze in Pflegeeinrichtungen in Echtzeit zur Darstellung gebracht. So konnten den behördlichen und politischen Entscheidungsträgern ad hoc Informationen aufbereitet zur Verfügung gestellt werden (Zusatzmaterial online: „Live-Daten zum Einsatzaufkommen in Pflegeeinrichtungen in FIRE Analytics", s. Hinweis am Anfang des Artikels).

\section{Bewältigung der Lage}

Gemäß der Fahrzeug- und Funktionsverteilung werden in der Berliner Notfallrettung im Tagdienst planmäßig 351 
Tab. 2 Übersicht der veröffentlichten Sonder-SOP und Aktualisierungen der Berliner Notfallrettung

\begin{tabular}{l|l|l|}
\hline Name & $\begin{array}{l}\text { Erstveröf- } \\
\text { fentlichung }\end{array}$ & $\begin{array}{l}\text { Versio- } \\
\text { nen }\end{array}$ \\
\hline $\begin{array}{l}\text { 1.1 Einsatzab- } \\
\text { lauf Notfallret- } \\
\text { tung }\end{array}$ & 24.01 .2020 & 24 \\
\hline
\end{tabular}

\subsection{Behand- \\ lungs- und \\ Zuweisungsstra- \\ tegie}

$17.03 .2020 \quad 5$

1.3 Intensivverlegung

\subsection{Mitnahme} von Begleitpersonen

1.5 Transportabschlussdesinfektion

1.6 Reanimation

$\begin{array}{ll}17.04 .2020 & 1\end{array}$

1.7 Risikobewer- $\quad 06.05 .2020 \quad 2$ tung

\subsection{Intubation}

3.1 Einsatzablauf NotSan-Erkunder

\subsection{Checkliste} NotSan-Erkunder

3.3 Dokumentation Telenotarzt

\subsection{Einsatzanla- ge Erkundung} Organisierte Wohnformen

3.5 AblaufErkundung Organisierte Wohnformen

\subsection{Meldewege}

4.2 Management von Kontaktpersonen

Die erste SOP. Beschreibt den Einsatzablauf für alle in der Notfallrettung Beteiligten (siehe $\bullet$ Abb. 4) mit allen Falldefinitionen und den daraus resultierenden Konsequenzen. Aufgrund der Fülle an Änderungen der RKI-Kriterien waren zeitweise tägliche Updates erforderlich

Beschreibt die Zuweisungsstrategie für Patienten mit Verdacht auf COVID-19-Erkrankung unter Würdigung des Patientenzustands und in Abstimmung mit dem SAVE-Berlin-Konzept

$19.03 .2020 \quad 3$

$24.03 .2020 \quad 2$

$14.04 .2020 \quad 3$

3

Regelt den Ablauf von Intensivverlegungen von der Anmeldung und Planung bis zur Durchführung im Rahmen des SAVE-Berlin-Konzepts

Änderung der Strategie zur Mitnahme von Begleitpersonen in Anbetracht der in den Kliniken geltenden Besuchsverbote und des möglichen Infektionsrisikos durch die Personen selbst

Regelung der Transportabschlussdesinfektion nach bestätigten COVID-19-Fällen

Schulungs-SOP zu Besonderheiten in der Reanimation im Kontext COVID-19 mit Hinweisen, beispielsweise zu Einsatzstellenmanagement, Umgang mit Ersthelfern oder Todesfällen

Weitere Schulungs-SOP, die die "awareness" der Einsatzkräfte gegenüber dem teilweise schwer vorhersehbarem, fulminanten Verlauf von COVID19 steigern soll. Mit Hinweisen zu "red flags" und Risikofaktoren

18.03.2020 4 Beschreibt Schritt für Schritt einen optimierten Ablauf für die Intubation mit Fokus darauf, eine Aerosolbildung so gering wie möglich zu halten

02.04.2020 $1 \quad$ Beschreibt den Einsatzablauf des neu aufgestellten Einsatzmittels NotSan-Erkunder und die verschiedenen Handlungsoptionen am Einsatzort

$02.04 .2020 \quad 1$

$02.04 .2020 \quad 2$

24.04.2020 1

$24.04 .2020 \quad 1$

$27.03 .2020 \quad 2$

$25.03 .2020 \quad 6$
Eine Checkliste für den Einsatz der NotSan-Erkunder, die auch die Indikation für den Einsatz des Telenotarztes regelt

Beschreibung der Dokumentation durch den Telenotarzt im Rahmen der gemeinsamen Einsätze mit den NotSan-Erkundern

Beschreibt die Möglichkeiten zur Identifikation von möglichen COVID-19-Hotspots in organisierten Wohnformen (z. B. Pflegeheimen)

Einsatz-SOP, die den Ablauf einer Erkundung in organsierten Wohnformen regelt

Eine "interne" SOP, die sämtliche Meldewege für bestätigte Fälle oder Verdachtsfälle sowohl bei Patienten als auch bei Mitarbeitern beschreibt

Umfangreiche SOP, die ebenfalls für den internen Gebrauch an der Mitarbeiter-Hotline erstellt wurde. Darin werden die Identifikation und das Management von Kontaktpersonen aller Kategorien geregelt
Funktionen (Einsatzkräfte) auf 172 Fahrzeugen und 78 Rettungswachen besetzt. Krankentransporte sind entsprechend dem Gesetz über den Rettungsdienst für das Land Berlin (RDG) nicht Teil der Notfallrettung, sondern werden privatwirtschaftlich organisiert durchgeführt. Insgesamt sind an den Aufgaben der Berliner Notfallrettung über 4000 Einsatzkräfte (rettungsdienstliches Fachpersonal) von insgesamt 12 unterschiedlichen Organisationen sowie zusätzlich über 500 akkreditierte Notärzte aus einem der 38 Berliner Notfallkrankenhäuser beteiligt.

Kennzeichnend für den gesamten Zeitraum der Pandemie war die hohe Dynamik, mit der sich die Rahmenbedingungen, das Einsatzgeschehen selbst, sowie die verfügbaren Informationen und Ressourcen veränderten. Eine zentrale Aufgabe über den gesamten Zeitraum der Pandemie bestand deshalb in der internen Krisenkommunikation. Im Zentrum standen dabei die fortlaufende Aktualisierung von SOP sowie die Aktualisierung von einsatztaktisch relevanten Informationen. Weiterhin waren Schwerpunkte die Vermittlung aktueller Erkenntnisse zu COVID-19-Erkrankungen sowie die Bedeutung von Hygieneund Schutzmaßnahmen.

Ein wichtiger Kanal der Informationsbündelung war darüber hinaus die tägliche Lagefortschreibung, in der zentral an alle Organisationen, Dienststellen und jede einzelne Einsatzkraft, der aktuelle Sachstand, wesentliche Neuerungen sowie die wichtigsten Informationen gebündelt per E-Mail durch den Einsatzstab der Feuerwehr herausgegeben wurden. Durch eine offene, ehrliche Kommunikation sollten den Mitarbeitenden Ängste genommen, Vertrauen geschaffen und der Verbreitung von Falschinformationen vorgebeugt werden. Kondensiert wurden diese Informationen - soweit für den Einsatzbetrieb unmittelbar von großer Bedeutung - auch auf den Wach-Displays (Bildschirmen auf allen Wachen), über Wachdurchsagen oder direkt in den Einsatzfahrzeugen zur Wahrnehmung gebracht.

Neben den täglichen Lagefortschreibungen und den internen Bulletins des Stabes für Führungskräfte wurden die 


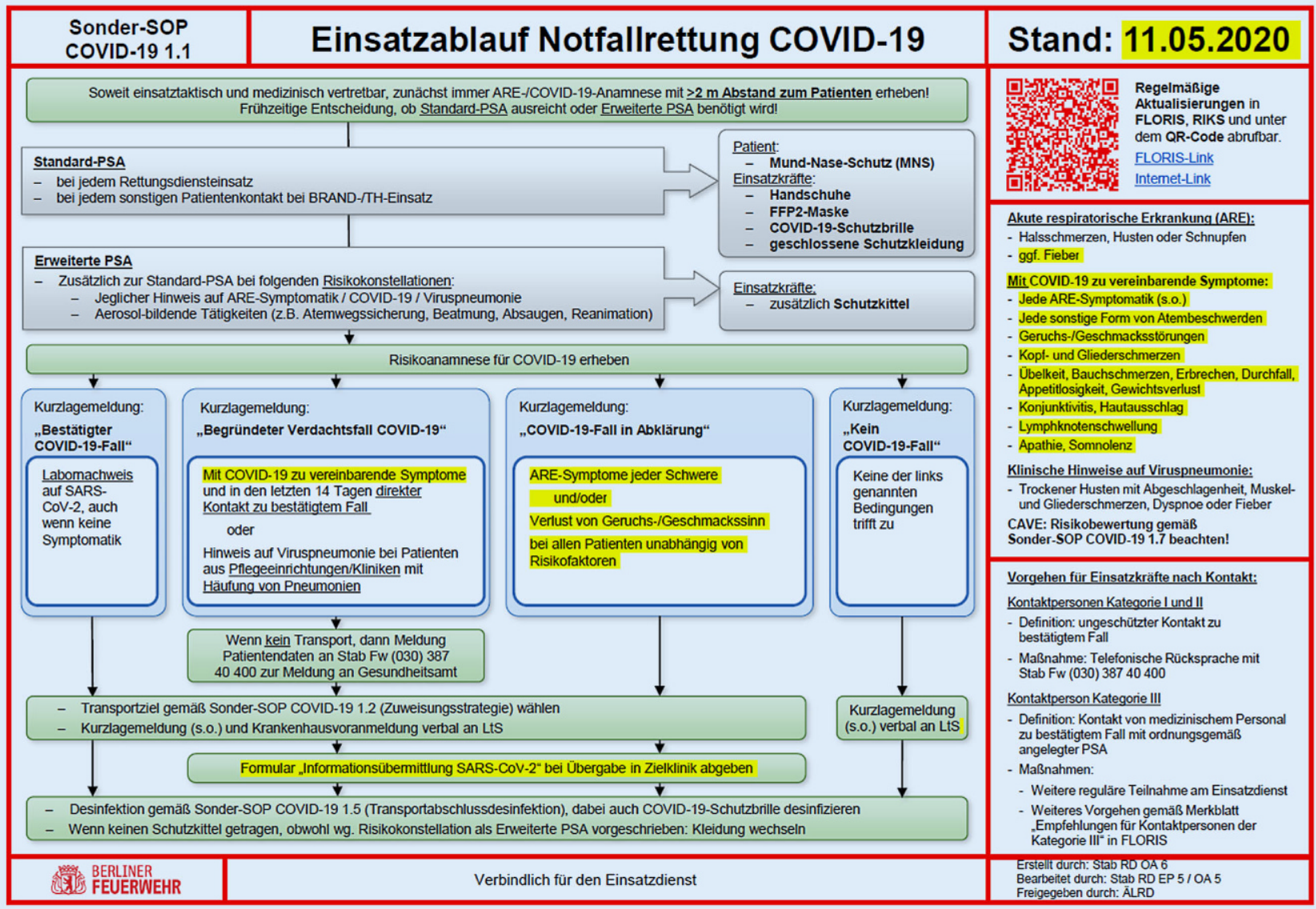

Abb. 4 ム Sonder-SOP COVID-191.1 Einsatzablauf Notfallrettung COVID-19. (Mit freundlicher Genehmigung der Berliner Feuerwehr). (SOP Standard Operating Procedures)

wichtigsten Entscheidungsträger täglich um 12 Uhr per Videokonferenz über das laufende Geschehen und erforderliche Entscheidungen ins Bild gesetzt. Für alle Fragen zum Thema Arbeitsschutz, Quarantänemaßnahmen und Umsetzung des Infektionsschutzgesetzes wurde eine rund um die Uhr erreichbare Hotline für alle Einsatzkräfte eingerichtet. Jenseits der internen Kommunikation wurde via Twitter, Facebook und YouTube die Öffentlichkeit über die Arbeit der Einsatzkräfte, aber auch alternative Ressourcen wie Sonderrufnummern oder allgemeine Verhaltenshinweisen informiert.

In der Berliner Notfallrettung sind alle medizinischen Handlungsanweisungen in SOP geregelt [8]. In der laufenden Pandemie wurden durch die ÄLRD Berlin 15 Sonder-SOP rund um den Umgang mit dem COVID-19-Ausbruch zusätzlich veröffentlicht (• Tab. 2) und insgesamt 58-mal aktualisiert.
Wichtigsten Baustein für die Einsatzkräfte stellte dabei die „SonderSOP-COVID-19 Einsatzdienst", die zentrale Handlungsanweisung für die Einsatzkräfte, dar (•Abb. 4), in der neben Hinweisen zu Schutzmaßnahmen alle wichtigen Informationen zur Fallklassifizierung, zum Verhalten der Einsatzkräfte an der Einsatzstelle und notwendige, durchzuführende Maßnahmen geregelt waren. Alle SOP wurden jeweils mit der täglichen Lagefortschreibung und frei zugänglich im Internet (https://www.berliner-feuerwehr.de/ service/mediathek/fachthemen/) veröffentlicht sowie auf den Wach-Displays und den iPads der Einsatzfahrzeuge zur Verfügung gestellt. Über einen QR-Code auf jeder Sonder-SOP konnte neben Versionsdatum durch die Anwendenden die Aktualität der vorliegenden Version überprüft werden.
Bereits in der Frühphase der pandemischen Ausbreitung von COVID-19 zeigte sich, dass es lageimmanent mehr und mehr zu Einschränkungen in der Regelversorgung kam. Während zunächst in zunehmender Anzahl Hausarztpraxen aufgrund von Personalausfall und fehlender Schutzausrüstung nicht mehr zur Verfügung standen [9], war auch die Versorgung durch den Ärztlichen Bereitschaftsdienst der Kassenärztlichen Vereinigung (ÄBD-KV) nicht mehr gewährleistet, da dieser im Land Berlin grundsätzlich über keine persönliche Schutzausrüstung (PSA) für den Einsatzdienst zur Versorgung infektiologischer Patienten verfügte [10]. Vor dem Hintergrund der wachsenden Zahl niedrigprioritärer Hilfeersuchen von Patienten mit ARE-Symptomen, die entsprechend der standardisierten Notrufabfrage eigentlich hätten an die KV-Leitstelle (116117) übergeben werden sollen, von 


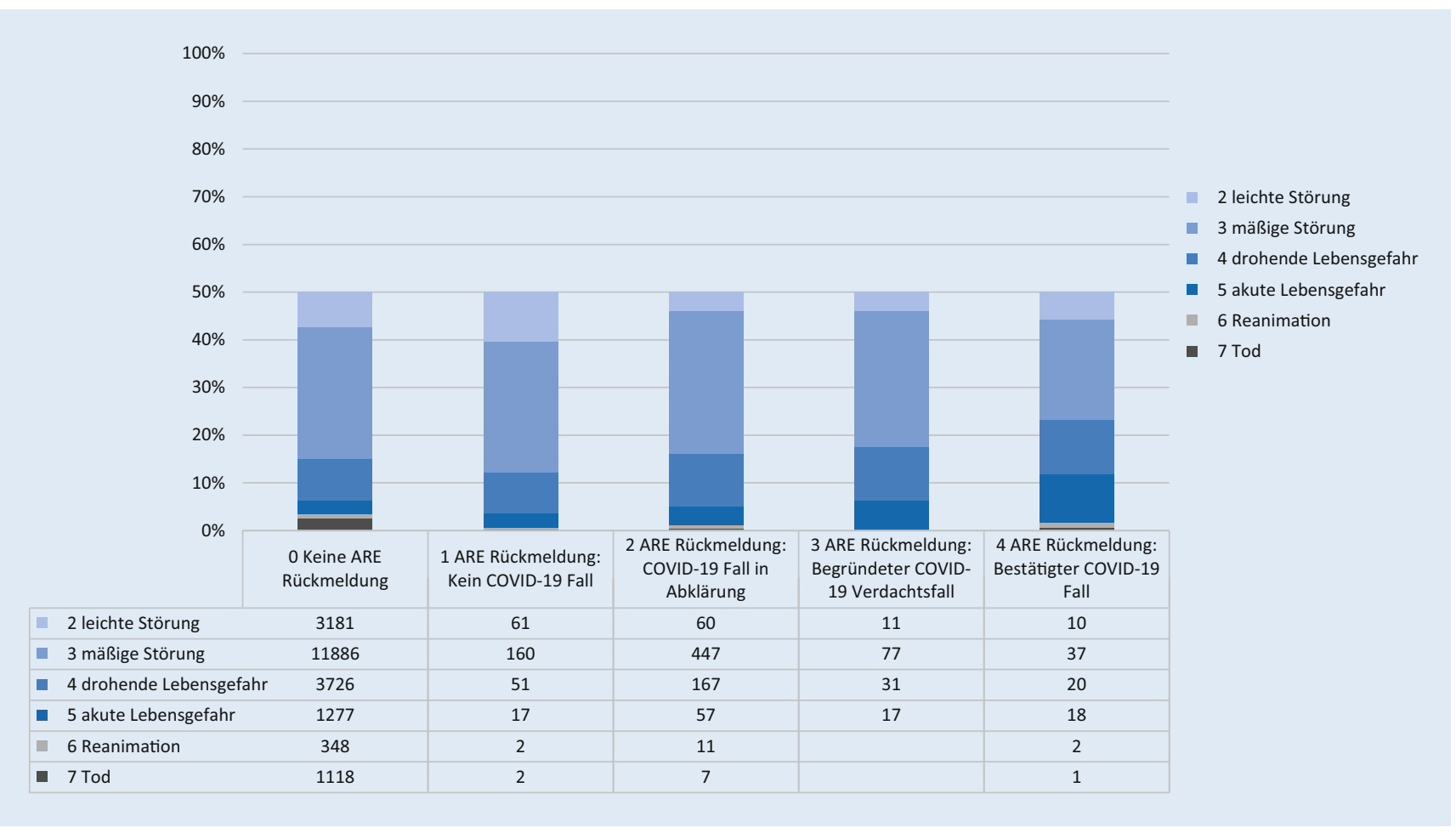

Abb. $5 \Delta$ Erkrankungsschwere der durch Notärzte versorgten ARE-Patienten gemäß NACA-Score. (NACA National Advisory of Aeronautics)

dort aber in Ermangelung verfügbarer Fahrzeuge bzw. PSA wieder zur Leitstelle der Berliner Feuerwehr (112) zurückvermittelt wurden, entschied sich die Berliner Feuerwehr in Ersatzvornahme Teile des ÄBD in Zusammenarbeit mit und von der KV zu übernehmen [10]. Täglich wurden somit 4 sog. KV-AREErkunder-Fahrzeuge durch die Berliner Feuerwehr in Betrieb genommen. Diese wurden von der Leitstelle der Berliner Feuerwehr disponiert und zu Einsätzen geschickt, die eigentlich durch den ÄBD hätten durchgeführt werden sollen. Die Fahrzeugführerfunktion wurde durch einen Mitarbeiter der Berliner Feuerwehr (Qualifikation mindestens Rettungssanitäter und langjährige Einsatzerfahrung in der Notfallrettung) gestellt; weiterhin wurde das Fahrzeug mit einem Arzt aus dem Personalpool des ÄBD der KV besetzt. Neben dem regulären Leistungsspektrum des ÄBD erfolgte zusätzlich die Ausstattung der KV-ARE-ErkunderTeams mit PCR-Abstrich-Sets, sodass auch systematische Testungen beispielsweise bei Ausbruchgeschehen in Gemeinschaftsunterkünften durch diese Teams möglich waren [11]. Insgesamt konnten im Zeitraum März bis Juni 2020 so bei 1262 Einsätzen der KVARE-Erkunder 1585 Patienten versorgt werden, von denen bei 1265 ein Abstrich durchgeführt werden musste. Die durchschnittliche Versorgung am Einsatzort dauerte aufgrund der aufwendigen hygienischen Vorsichtsmaßnahmen sowie der erforderlichen Sorgfalt bei einer sehr heterogenen Beschwerdelage im Mittel 40 min. Zusätzlich zu den KVARE-Erkundern der Berliner Feuerwehr wurde durch die KV die hausärztliche Beratungsleistung in der Leitstelle der 116117 intensiviert, um - soweit fachlich möglich - Hilfeersuchen rein telefonisch abzuarbeiten.

Als besondere Herausforderungen von COVID-19 zeigte sich insbesondere die teilweise schwierige telefonische Triagierung der an COVID-19 erkrankten Patienten, zum einen aufgrund der unspezifischen Symptomlage und zum anderen aufgrund der teilweisen rapiden Verschlechterung des Zustands einiger Patientinnen und Patienten innerhalb weniger Stunden. Vor diesem Hintergrund musste auch eine große Anzahl der Patienten mit moderater
Krankheitslast zur differenzialdiagnostischen Entscheidungsfindung im Notarztdienst versorgt und überwiegend in ein Krankenhaus transportiert werden (• Abb. 5). Um zu vermeiden, dass sämtliche Patienten bereits schon mit milden Symptomen in eine Klinik transportiert werden mussten, und zur Anbindung an alternative Versorgungsformen zum Krankenhaus wurden 4 weitere Einsatzfahrzeuge als „NotSan-Erkunder“ in Dienst genommen, die mit einem NotSan-Auszubildenden als Fahrer und einem besonders erfahrenen Notfallsanitäter der BFRA besetzt waren. Aufgabe der NotSan-Erkunder war es, innerhalb kurzer Zeit mit ihrem Fahrzeug Patienten aufzusuchen, deren Hilfeersuchen gemäß Notrufabfrage ansonsten, wenn auch weniger prioritär, mit einem RTW beschickt worden wären. Am Einsatzort wurde durch die NotSan-Erkunder eine systematische Untersuchung anhand einer standardisierten Checkliste durchgeführt. Bei kritischen Patienten oder bei Patienten mit einer stationären Behandlungsbedürftigkeit erfolgte die unmittelbare Nachalarmierung der erforderlichen Einsatzmittel (RTW und 


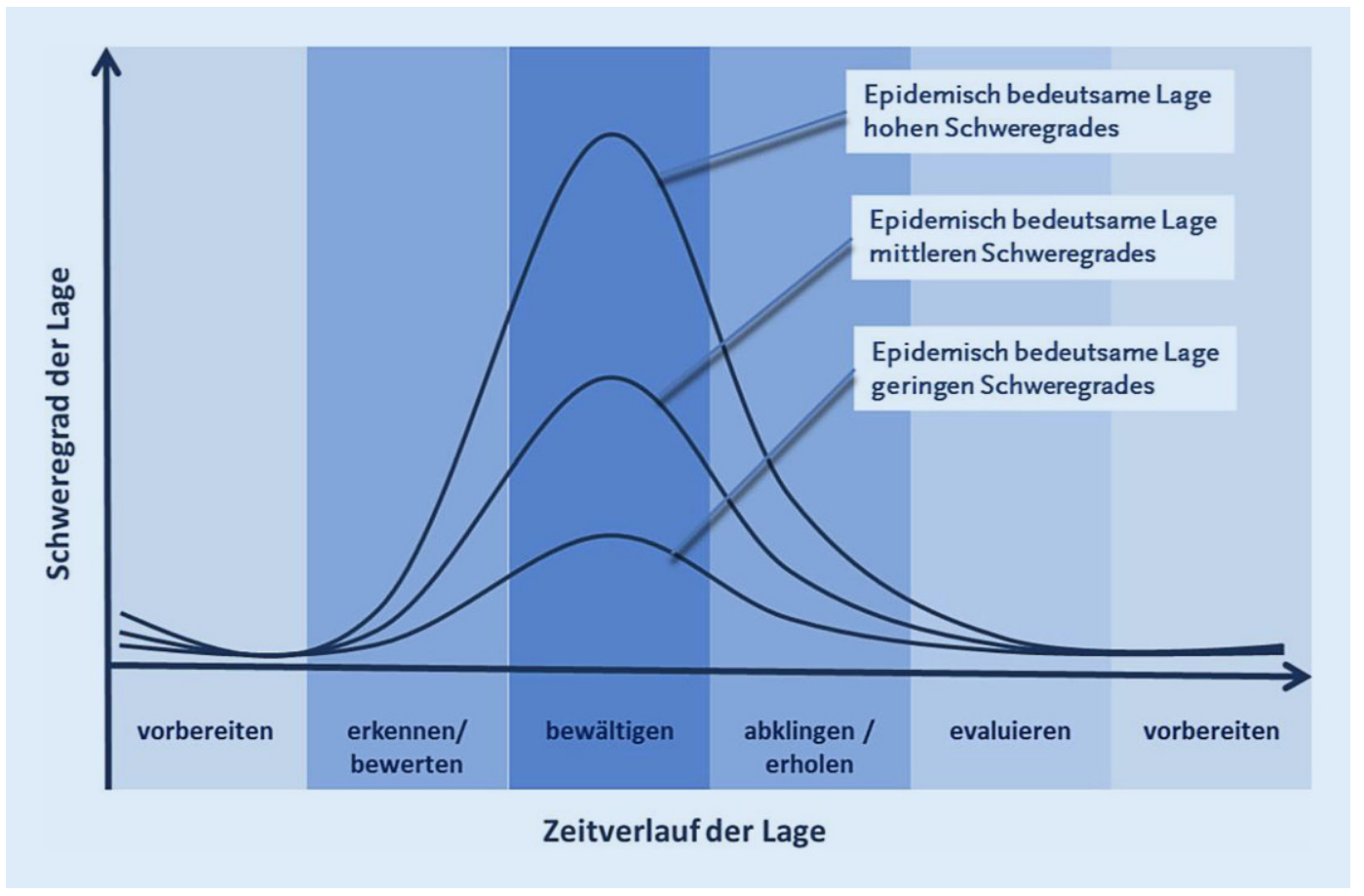

Abb. $6<$ Schematischer Überblick des RKI zu Ausmaß (Schweregrad) und Phasen (Zeitverlauf) von epidemiologisch bedeutsamen Lagen biologischen Ursprungs. (Nach Robert Koch-Institut [13]) ggf. NEF); bei Patienten, deren Gesundheitszustand und Versorgungssituation eine ambulante Versorgung zuließen, erfolgten nach telefonischer Abstimmung mit dem Telenotarzt eine erweiterte Aufklärung und nach Möglichkeit die Entscheidung zu einer ambulanten Versorgung oder den Patienten an andere Versorgungsformen beispielsweise eine Follow-up-Visite durch eines der KVErkunder ARE-Teams zu vermitteln. Während der Hochphase der ersten Welle der Pandemie konnten im April 2020 bei 341 Einsätzen durch NotSanErkunder $49 \%$ aller Patienten anstelle eines Krankenhauses an alternative Versorgungsangebote angebunden werden [12].

Neben der individuellen Patientenversorgung wurden das Einsatzmittel NotSan-Erkunder gemeinsam mit dem OAvD außerdem zu Ausbruchsgeschehen in organisierten Wohnformen zur Erkundung mit entsprechender Triagierung und Versorgung der Bewohner eingesetzt.

\section{Gewonnene Erkenntnisse}

Grundsätzlich konnten bei der andauernden Lagebewältigung zu COVID-19 im Bereich der Notfallrettung im Land Berlin medizinische, organisatorische und technische sowie die verfügbaren Ressourcen betreffende Herausforderungen unterschieden werden (- Tab. 3). Schweregrad und Zeitverlauf der COVID-19Pandemie folgten dabei dem vom RKI beschriebenen typischen Verlauf von epidemiologisch bedeutsamen Lagen (• Abb.6, nach [13]).

Insgesamt zeigten sich Herausforderungen im Zusammenhang mit COVID19 insbesondere überall dort, wo auch im Alltag bereits Verbesserungspotenzial hinsichtlich Zuständigkeiten, Strukturen und Prozessen von den beteiligten Akteuren identifiziert war. Durch die besondere Tragweite, Intensität und Dynamik der epidemiologischen Entwicklungen kamen teilweise bekannte Schwächen besonders deutlich zum Tragen. Wenn gleichwohl kaum ein Bereich unserer Gesellschaft nicht unmittelbar und umfassend von den Auswirkungen von COVID-19 betroffen ist, so kann zweifellos festgestellt werden, dass die Notfallrettung als Teil der Gesundheitsund Sozialsysteme in Deutschland besonders stark und in erster Linie an der Kompensation und Bewältigung der Auswirkungen beteiligt ist. Gleichsam einem roten Faden wurde immer dann deutlich, dass die Notfallrettung „letzte Wiese“ der Versorgung und struktureller
Verantwortung ist, wenn andere - primär zuständige - Versorgungsstrukturen versagten. Notfallrettung und Notfallmedizin bilden damit unterhalb der Katastrophe das entscheidende Rückgrat für die Resilienz unserer Gesellschaft und die Akzeptanz der Funktionsfähigkeit von unmittelbarer Daseinsführsorge. Notfallrettung geht in Letztzuständigkeit und Leistungsspektrum weit über eine Lotsenfunktion in der Leitstelle der 112 oder die Abwendung einer unmittelbaren Gefahr für Leib oder Leben hinaus. Über die Bewältigung der aktuellen Herausforderungen von COVID-19 hinaus wird es Gegenstand laufender Gesetzgebungsverfahren sein, dieser faktischen Rolle des erweiterten und ultimativen Sicherstellungsauftrags der Notfallrettung in einem qualitativ und quantitativ gewachsenen Aufgabenspektrum unserer Gesundheitssysteme Rechnung zu tragen [14].

Die gewonnenen Erkenntnisse in der Berliner Notfallrettung decken sich mit den Erfahrungen anderer internationaler Rettungsdienstorganisationen. So konnten Ventura et al. Herausforderungen durch einen erheblichen Mangel erforderlicher Schutzausrüstungen und bestehende Unsicherheiten im Umgang mit COVID-19-Patienten im Bereich 
Tab. 3 Identifizierte Herausforderungen und Antworten bei der Lagebewältigung

Medizinisch Wiederholt wechselnde Falldefinitionen/-klassifikationen

$\Rightarrow$ permanentes Monitoring und Bewertung aller zur Verfügung stehenden wissenschaftlichen Fachinformationen zur Abwägung von Kontinuität und notwendiger Änderungen zugunsten erhaltender Kompatibilität durch mehrere zuständige Oberärzte der Ärztlichen Leitung des Rettungsdienst

Schneller Wandel hinsichtlich empfohlener Maßnahmen (Schutzmaßnahmen/Therapie)

$\Rightarrow$ kontinuierliche, regionale Übersetzung des geltenden Standes der medizinischen Wissenschaft durch die Ärztliche Leitung des Rettungsdienstes

Schwierige Einschätzung der Erkrankungsschwere und Dynamik

$\Rightarrow$ starke Ausweitung der überwiegend telefonischen Beratung und Supervision des Einsatzgeschehens durch den diensthabenden OAvD. Entwicklung eines digitalen Wächter-Tools bei wiederholten (ARE.-)Einsätzen zur gleichen Einsatzörtlichkeit

Schwieriges Monitoring von Risikogruppen, insbesondere organisierten Wohnformen/Obdachlosigkeit

$\Rightarrow$ Entwicklung eines digitalen Analyse-Tools und automatisierten Reportings zu Anzahl und Häufungen von Einsätzen und Einsatzanlässen in Einrichtungen je Bewohner/Bett

Organisa- Große Dezentralität und Heterogenität im öffentlichen Gesundheitsdienst (ÖGD) und bei der Umsetzung des Infektionsschutzgesetzes torisch (IFSG)

$\Rightarrow$ mindestens wöchentliche, teilweise tägliche Telefon- oder Videokonferenzen mit allen regionalen Akteuren des Gesundheits wesens, insbesondere dem ÖGD

Begrenzte Leistungs- und Aufwuchsfähigkeit alternativer Versorgungsangebote (116117, Pflegedienste, psychiatrische Versorgungsangebote)

$\Rightarrow$ bleibende Herausforderungen mit konsekutiver Ersatzvornahme der Berliner Notfallrettung überall dort, wo andere Strukturen oder Angebote nur noch eingeschränkt oder gar nicht mehr zur Verfügung standen

Unterschiedliche Ausstattung/Ausrüstung der beteiligten Organisationen der Notfallrettung

$\Rightarrow$ Umstellung in den Mangelressourcen von einer dezentralen, organisationsbezogenen auf eine landesweite, gemeinsame Beschaffung aller am Gesundheitswesen beteiligten Akteure

Akzeptanz erforderlicher Hygienemaßnahmen und Schutzausrüstung in der Frühphase der Pandemie

$\Rightarrow$ kontinuierliches Veränderungsmanagement durch Information, Schulung und wiederkehrende Sensibilisierung

Technisch Erreichungsgrad aller Einsatzkräfte bei hoher Informationsdichte und geringer Halbwertszeit

$\Rightarrow$ Systematisierung und Konzentration der Kommunikationswege auf einige wenige kontinuierlich genutzter Kanäle nach dem Prinzip „low dose - high frequency" (täglicher Lagebricht, Wachdurchsagen zum Schichtwechsel, Videobotschaften)

Umstellung aller regulären Besprechungsformate auf Videokonferenzen

$\Rightarrow$ umfangreiche Investition in Videokonferenztechnik und Software-Lizenzen, Telefonweiterleitungskapazitäten, VPN-Technik

Umstellung aller Schulungskonzepte auf Webinare

$\Rightarrow$ Entwicklung umfangreicher Blended-Learning-Curricula für bestehende Aus- und Fortbildungsformate

Wiederholtes Zusammenbrechen der 116117 wegen unzureichender Telekommunikationsinfrastruktur und konsekutivem Anstieg der Last auf der 112

$\Rightarrow$ Ausbau der Leistungsfähigkeit der Telekommunikationsinfrastruktur bei der KV und zur Kompensation in der integrierten Leitstelle der Berliner Feuerwehr, einschließlich der Vorbereitung vollwertiger Redundanzen

Verfügbare

Unzureichende Lagerbestände/Reserven bei Verbrauchsmaterialien (On-Demand-Versorgung)

$\Rightarrow$ Vorratshaltung von 3 Monaten bei allen Verbrauchsmaterialien, einschließlich Medikamenten

Unzureichende Anzahl verfügbarer Schutzausrüstung

$\Rightarrow$ umfangreicher Aufbau erheblicher Lagerbestände für Schutzausrüstung, gemessen am aktuellen Verbrauch

Fehlende Testmöglichkeiten und Laborverträge für Einsatzpersonal und Patienten

$\Rightarrow$ Aufbau einer eigenen Teststrecke für das Einsatzpersonal in Zusammenarbeit mit der Polizei Berlin sowie für den Test von Patient*innen mit dem Labor Berlin

Starke Kompetition um insbesondere notärztliches Personal (Notärzte für ITW) mit den Kliniken, da dort derselbe Personenkreis für die Intensivstationen gebraucht wird

$\Rightarrow$ Frühzeitige Kommunikation des Personalsonderbedarfs auf Basis flächendeckend bestehender Notarztverträge mit den Berliner Krankenhäusern; im Rahmen dessen auch, soweit möglich, Separieren unterschiedlicher Dienstgruppen zu Unterbrechung und Redundanz bei Infektionsketten

verschiedener Rettungsdienste in den USA feststellen [15]. Fernandez et al. konnten zeigen, dass mit den dem Rettungsdienst zur Verfügung stehenden Mitteln eine sichere Identifikation von COVID-19-Patienten nur unzureichend möglich ist und deshalb z. B. hinsichtlich der Schutzbekleidung grundsätzlich bei allen Einsätzen von einem infektiologischen Risiko ausgegangen werden muss; dies deckt sich mit dem Vorgehen in den Berliner SOP [16]. Anders als Lai et al. für den Rettungsdienst in New York festgestellt haben, konnte während der andauernden COVID-19-Pandemie in der Berliner Notfallrettung kein Anstieg an Reanimationen registriert werden [17]. Auch der von Lerner et al. in den USA beschriebene, zwischenzeitlich starke Rückgang der Einsatzzahlen des Rettungsdienstes ist in Berlin deutlich flacher ausgefallen [18] und entspricht eher dem von Hagebusch et al. für die Stadt Frankfurt a. M. beschriebenen 
Umfang anderer deutscher Großstädte [19]. Sechi et al. hingegen beschrieben, dass, wie in Berlin auch, in der Lombardei die Einführung von „business intelligence (BI) tools“ in der Leitstelle und im Rettungsdienst entscheidend zur Bewältigung der Herausforderungen der Pandemie beigetragen hat [20]. Auch die von Sayers et al. geforderte Nutzung von aggregierten Daten des Rettungsdienstes für (gesundheitspolitische) Entscheidungsträger konnte mit dem VOST-Team der Berliner Feuerwehr bereits umgesetzt werden [21]. Die Bedeutung der Einführung von ambulanten COVID-19-Testkapazitäten des Rettungsdienstes zur Vermeidung unnötiger Krankenhausvorstellung vulnerabler Patientengruppen, so wie sie in Berlin umgesetzt wurde, deckt sich ferner mit den durch Goldberg et al. für den Rettungsdienst in Boston beschriebenen Erfahrungen [22].

\section{Fazit für die Praxis}

- COVID-19 stellt eine außerordentliche Belastungsprobe für das Gesundheitswesen insgesamt und die notfallmedizinische Versorgung im Besonderen dar.

- Die Notfallrettung übernimmt die entscheidende Rolle der ambulanten Notfallversorgung und präklinischen Patientensteuerung insbesondere auch dort, wo andere Versorgungsangebote nicht oder nur noch eingeschränkt zur Verfügung stehen.

- Die Bewältigung von epidemiologisch bedeutsamen Lagen in der Notfallmedizin erfordert eine frühzeitige, systematische Lageüberwachung, eine detaillierte, digitale Lagedarstellung und eine agile, an regionale Gegebenheiten angepasste Lagebewältigung.

- Neue Einsatzmittel insbesondere im Bereich niedrig prioritärer Hilfeersuchen können helfen, den besonderen Herausforderungen einer Pandemie besser zu begegnen.

- Die hohe Dynamik epidemiologischer Entwicklungen erfordert kontinuierliche, systematische Kommunikation und stets aktualisierte, praktische, medizinische Handlungsempfehlungen (SOP).

\section{Korrespondenzadresse}

Dr. med. Janosch Dahmen

Fakultät für Gesundheit, Department

Humanmedizin, Universität Witten/Herdecke

Alfred-Herrhausen-Straße 50, 58448 Witten,

Deutschland

janosch.dahmen@uni-wh.de

Funding. Open Access funding enabled and organized by Projekt DEAL.

\section{Einhaltung ethischer Richtlinien}

Interessenkonflikt. J. Dahmen, L. Bäker, F. Breuer, K. Homrighausen, C. Pommerenke, J.-K. Stiepak und $S$. Poloczek geben an, dass kein Interessenkonflikt besteht.

Für diesen Beitrag wurden von den Autoren keine Studien an Menschen oder Tieren durchgeführt. Für die aufgeführten Studien gelten die jeweils dort angegebenen ethischen Richtlinien.

Open Access. Dieser Artikel wird unter der Creative Commons Namensnennung 4.0 International Lizenz veröffentlicht, welche die Nutzung, Vervielfältigung, Bearbeitung, Verbreitung und Wiedergabe in jeglichem Medium und Format erlaubt, sofern Sie den/die ursprünglichen Autor(en) und die Quelle ordnungsgemäß nennen, einen Link zur Creative Commons Lizenz beifügen und angeben, ob Änderungen vorgenommen wurden.

Die in diesem Artikel enthaltenen Bilder und sonstiges Drittmaterial unterliegen ebenfalls der genannten Creative Commons Lizenz, sofern sich aus der Abbildungslegende nichts anderes ergibt. Sofern das betreffende Material nicht unter der genannten Creative Commons Lizenz steht und die betreffende Handlung nicht nach gesetzlichen Vorschriften erlaubt ist, ist für die oben aufgeführten Weiterverwendungen des $\mathrm{Ma}$ terials die Einwilligung des jeweiligen Rechteinhabers einzuholen.

Weitere Details zur Lizenz entnehmen Sie bitte der Lizenzinformation auf http://creativecommons.org/ licenses/by/4.0/deed.de.

\section{Literatur}

1. European Emergency Medical Services Leadership Network https://emsleadershipnetwork.org. Zugegriffen: 1. Juli 2020

2. WHO (Hrsg) Weltgesundheitsorganisation WHO zu neuartigem Virus am 05. Januar 2020. https://www.who.int/csr/don/05-january-2020pneumonia-of-unkown-cause-china/en/. Zugegriffen: 1. Juli 2020

3. WHO (Hrsg) US-Nachrichtenplattform zu Infektiologischen Ausbruchgeschehen in Asien am 05. Januar 2020. https://www.statnews.com/2020/ 01/05/cause-of-mysterious-pneumonia-cases- still-unknown-chinese-say/. Zugegriffen: 1. Juli 2020

4. BayrischerRundfunkzurersten COVID-19Infektion in Deutschland. https://www.br.de/nachrichten/ bayern/coronavirus-webasto-schliesst-standortstockdorf-voruebergehend, RouGdts. Zugegriffen: 1. Juli 2020

5. Luiz T, Marung H, Pollach G et al (2019) Implementierungsgrad der strukturierten Notrufabfrage in deutschen Leitstellen und Auswirkungen ihrer Einführung. Anaesthesist 68:282-293. https://doi. org/10.1007/s00101-019-0570-6

6. International Academies of Emergency Dispatch (IAED) Protokoll 36 zu Pandemie/Epidemie/ Ausbruch. https://cdn.emergencydispatch.org/ iaed/pdf/NAE-Protocol-36-Flu-v13_3.pdf. Zugegriffen: 1. Juli 2020

7. International Academies of Emergency Dispatch (IAED) Protokoll 36 zu Pandemie/Epidemie/ Ausbruch. https://cdn.emergencydispatch.org/ iaed/pdf/NAE_Pandemic_v13-3.pdf.Zugegriffen 1.Juli 2020

8. Standard Operation Procedures (SOP) der Ärztlichen Leitung Rettungsdienst (ÄLRD) für die Berliner Notfallrettung. https://www.berlinerfeuerwehr.de/service/mediathek/fachthemen/. Zugegriffen: 1. Juli 2020

9. Rundfunk Berlin Brandenburg Abendschau, 25.03.2020, 19.30 Uhr. https://www.rbb24. de/panorama/beitrag/2020/03/100-arztpraxengeschlossen-berlin-corona-.html. Zugegriffen: 1. Juli 2020

10. Tagesspiegel zu Einschränkungen der Versorgung im ärztlichen Bereitschaftsdienst (ÄBD). https://www.tagesspiegel.de/berlin/wir-werdenvoellig-allein-gelassen-berliner-hausaerztestellen-sich-auf-das-coronavirus-ein/25606768. html. Zugegriffen: 1. Juli 2020

11. Pressemitteilung Kassenärztliche Vereinigung Berlin zur Sicherstellung des Ärztlichen Bereitschaftsdienst für COVID-19-Patienten durch die Berliner Feuerwehr. https://www.kvberlin. de/40presse/10pressemitteilung/pe200303.html. Zugegriffen: 1. Juli 2020

12. Breuer F, Poloczek S, Dahmen J et al (2020) Einführung von NotSan-Erkundern im Rahmen der COVID-19 Pandemie in der Berliner Notfallrettung. Notfall Rettungsmed. https://doi.org/10.1007/ s10049-020-00786-5

13. Robert Koch-Institut (RKI) Rahmenkonzept mit Hinweisen Für Medizinisches Fachpersonal und den öffentlichen Gesundheitsdienst in Deutschland: Epidemisch bedeutsame Lagen erkennen, bewerten und gemeinsam erfolgreich bewältigen 2020, Seite 3. https://www.rki.de/DE/Content/Infekt/ Preparedness_Response/Rahmenkonzept_ Epidemische_bedeutsame_Lagen.pdf; jsessionid=4FD0E9A70BA32214A3A2212DAD 924EDD.internet082?_blob=publicationFile. Zugegriffen: 1. Juli 2020

14. Bundesministerium für Gesundheit (BMG) Referentenentwurf zum Gesetz zur Reform der Notfallversorgung. https:// www.bundesgesundheitsministerium.de/ notfallversorgung.html.Zugegriffen: 1. Juli 2020

15. Ventura C, Gibson C, Collier GD (2020) Emergency Medical Services resource capacity and competency amid COVID-19 in the United States: preliminary findings from a national survey. Heliyon 6(5):e3900. https://doi.org/10.1016/j.heliyon. 2020.e03900

16. Fernandez AR, Crowe RP, Bourn S et al (2020) COVID-19 preliminary case series: characteristics 
of EMS encounters with linked hospital diagnoses. Prehosp Emerg Care. https://doi.org/10.1080/ 10903127.2020.1792016

17. Lai PH, Lancet EA, Weiden MD et al (2020) Characteristics associated with out-of-hospital cardiac arrests and resuscitations during the novel Coronavirus disease 2019 pandemic in New York city. JAMA. https://doi.org/10.1001/jamacardio. 2020.2488

18. Lerner EB, Newgard CD, Mann NC (2020) Effect of the Coronavirus disease 2019 (COVID-19) pandemic on the U.S. emergency medical services system: a preliminary report. Acad Emerg Med. https://doi.org/10.1111/acem.14051

19. Hagebusch P, Naujoks F, Rouchi $\mathrm{H}$ et al (2020) Decline in emergency medical service missions during the COVID-19 pandemic: results from the fifth largest city in Germany. Intern Emerg Med. https://doi.org/10.1007/s11739-020-02482-1

20. Sechi GM, Migliori M, Dassi G et al (2020) Business Intelligence applied to Emergency Medical Services in the Lombardy region during SARSCoV-2 epidemic. Acta Biomed 91(2):39-44. https:// doi.org/10.23750/abm.v91i2.9557

21. Goldberg SA, Bonacci RA, Carlson LC, Pu CT, Ritchie CS (2020) Home-based testing for SARScoV-2: leveraging prehospital resources for vulnerable populations. West J Emerg Med 21(4):813-816. https://doi.org/10.5811/westjem. 2020.5.47769

22. Sayers DR, Hulse ST, Webber BJ, Burns TA, Denicoff AL (2020) Notes from the field: use of emergency medical service data to augment COVID-19 public health surveillance in montgomery county, maryland, from march to June 2020. JMIR Public Health Surveill 6(3):e22331. https:// doi.org/10.2196/22331

\section{In eigener Sache}

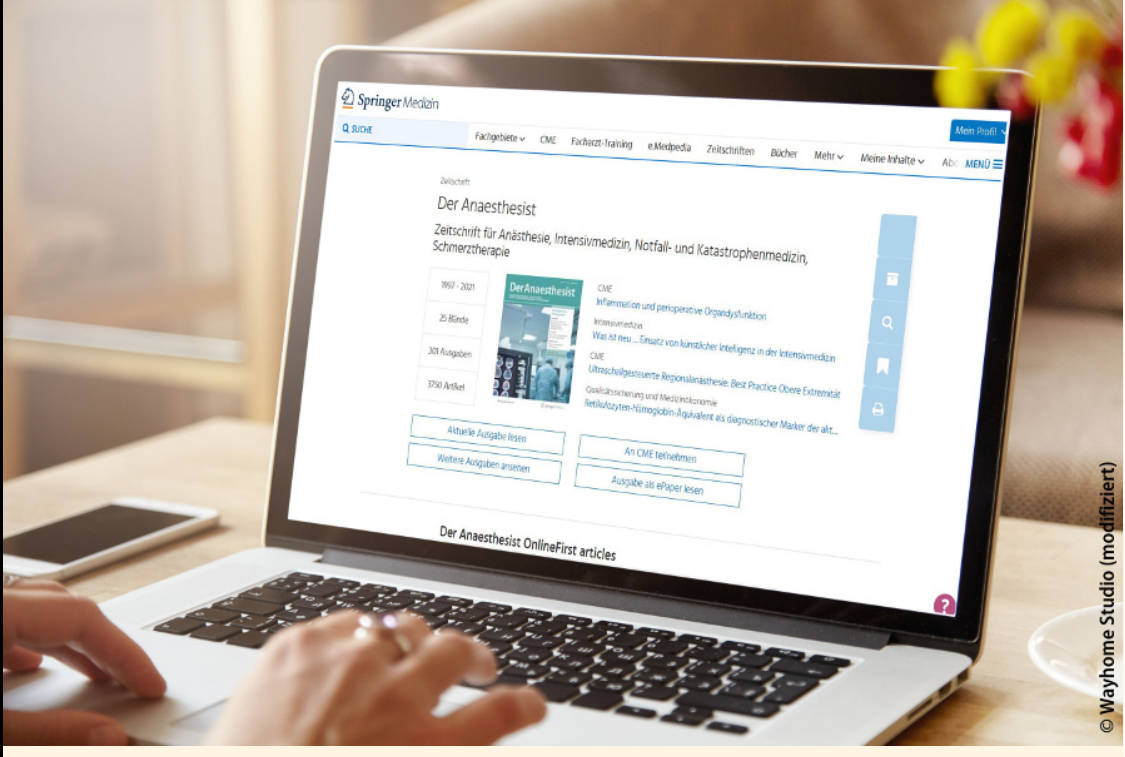

\section{Lesen Sie online weiter!}

Wussten Sie, dass Sie als Abonnent*in einer Zeitschrift automatisch auch online Zugriffsrechte auf das gesamte Beitragsarchiv haben?

Durch Ihr Abonnement von Der Anaesthesist erhalten Sie jeden Monat die aktuelle Ausgabe der Zeitschrift nach Hause geliefert. Doch damit nicht genug: Sie haben mit dem Abonnement außerdem Zugriff auf das gesamte Online-Archiv Ihrer Zeitschrift.

Und so einfach geht es:

Registrieren Sie sich einmal über www.springermedizin.de/register:

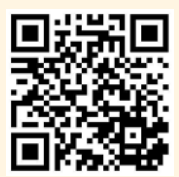

Über diesen QR-Code schnel und einfach registrieren

Bei der Registrierung geben Sie einfach Ihren Vor- und Nachname und Lieferadresse wie beim Abonnement der Zeitschrift (siehe Adressaufkleber auf Ihrem Heft) an. So kann im System die Zugehörigkeit zu Ihrer Zeitschrift sichergestellt werden.

Sollten Fragen oder Probleme auftauchen, wenden Sie sich einfach an den Kundenservice:

kundenservice@springermedizin.de
Aufgrund des Heilmittelwerbegesetzes dürfen die Inhalte der Website nur medizinischen Fachkreisen zur Verfügung gestellt werden. Bei der Anmeldung bitten wir Sie deshalb einen Berufsnachweis vorzulegen. Bei Mediziner*innen mit Mitgliedschaft in der deutschen Ärztekammer reicht die einheitliche Fortbildungsnummer (EFN). Als Angehörige*r eines medizinischen Berufs schicken Sie eine Bestätigung der Arbeitsstelle. Sind Sie Student*in, dann bitte den Studiennachweis mit Angabe des Studiengangs ganz unkompliziert an kundenservice@springermedizin.de.

Mit Benutzername und Passwort haben Sie außerdem Zugang zu den freien Inhalten auf den Seiten von: https://www.springermedizin.de/ https://www.aerztezeitung.de/ 